\section{T. 16208}

PPPL-2115

$D R=0288.5$

PORTIONS OF THIS AEPORT ARE ILLEGIELE IT

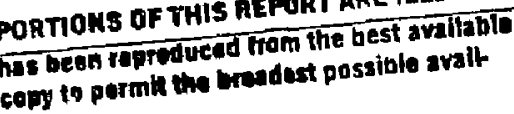

alitity.

TRAPPED PARTICLE DESTABILIZATION OF THE INTERNAL KINK MODE

By

R.B. White, L. Chen, F. Romane111, and R. Hay

JUNE 1984
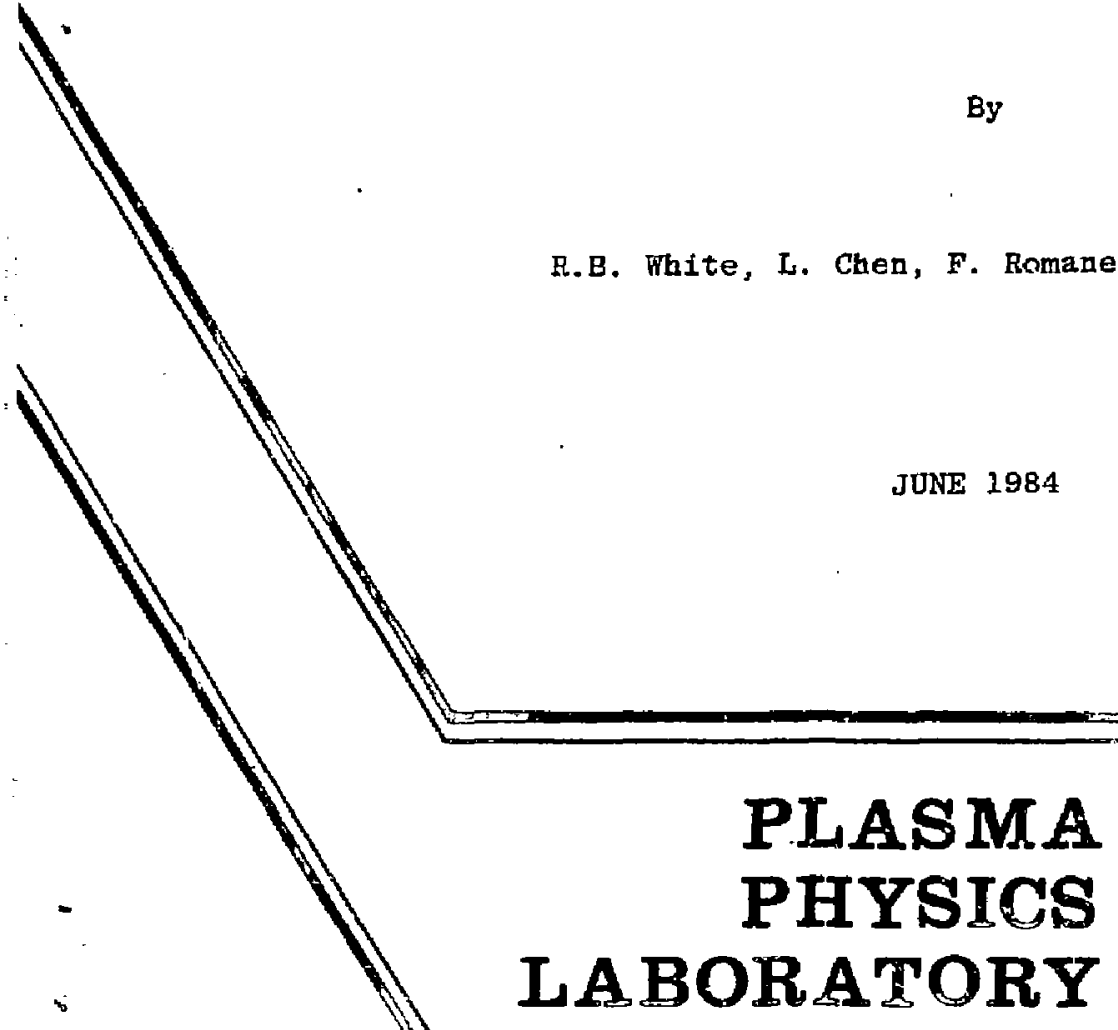

PLASMA

PHYSIGS

MASTER

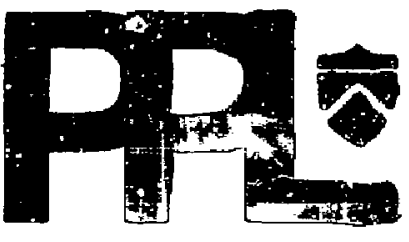

PRINCETON UNIVERSITY

PRINCETON, NEW JPRSEY

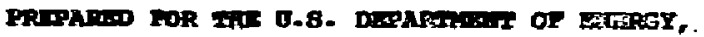

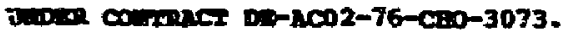


This zeport was grepared as an aceount of werk sponsored by the

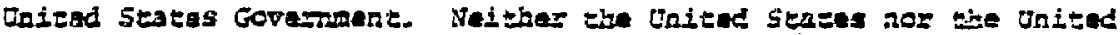

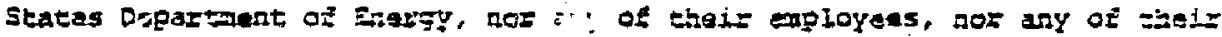
contrareors, subcenzacters, or thair edoloyees, maikes any war=anty, oxprass or inplied, or assure's any logal jibilitey or responsibilisy Eor

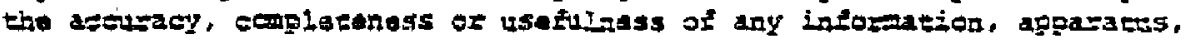
procuct or jrocsss disclosed, or zaprasents that its use would not infinge Drtoately owned rights.

Printed is the United States of huerica.

\section{Arailabie 5 mom:}

National Technical Intoration Servire

J. S. Deparzent of Cermerze 3285 Dort keyal jodd

Sgriagrield, Virginia 22151

Frice. Printed Copy \$_._._. Wicrorische $\$ 3.50$

DAGES

$i-25$

$26-50$

$51-75$

$76-100$

$10 ?-125$

$126-150$

$151-175$

$176-200$

$201=225$

$226-250$

$251-275$

$276-300$

$301-325$

$326-3 \equiv 0$

$35 I-3 \% 5$

$376-400$

$401-425$

425-450

$451-475$

$476-500$

$500-\$ 25$

$526-350$

$551-575$

$575-500$
NIS

Selingr Reice

$\$ 5.00$

$\$ 6.50$

$\$ 9.00$

$\$ 9.50$

\$II. 00

$\$ 12.50$

$\$ 14.00$

$\$ 13.50$

$\$ 17.00$

$\$ 19.50$

$\$ 20.00$

$\$ 21.50$

$\$ 23.00$

$\$ 24.50$

$\$ 26.00$

$\$ 27.50$

$\$ 29.00$

$\$ 30.50$

$\$ 32.00$

$\$ 33.50$

$\$ 35.00$

$\$ 36.50$

$\$ 38.00$

$\$ 39.50$

\author{
Fos docmenes over 600 \\ pages, add $\$ 1$. .0 इor pach \\ additional is page inczament.
}




\author{
R. B. White, L. Chen, F. Romanelli, + and R. Hay \\ Plasma Physics Laboratory, Princeton University \\ P.O. BOX 451 \\ Princeton, New Jersey 0B544
}

PPPL --2115

DE84 015150

\begin{abstract}
The internal kink mode is destabilized by trapped high energy particles, leading to a new branch of the internal kink disfersion relation with a real Erequency near the average trapped particle precession frequency and a growth rate of the same magnitude. This trapped particle branch of the dispersion relation is investigated numerically for a variety of particle distributions. Made growth rate and frequency is found as a function of plasma $B$, density, and trapped particle energy and distribution. The high energy trapped particle sources considered are neutral bean injection, ion cyclotron heating, and fusion alpha particles. Relevance for vasious plasma heating schemes is disclissed.
\end{abstract}

\title{
DISClaIMER
}

\begin{abstract}
This repont was prepared as an aceount of work sponsored by an agency of the United State Goverament. Neither the United Stz:es Government nor any agency thereof, nor any of their employees, makes any warranty, express or implied, or assumes any legal liability or responsibility for the accuracy, completeness, or usefulness of any information, apparatus, product, or process disclosed, or represents that is use would not infringe privately owned rights. Reference herein to any specific commercial product, process, or service by trade name, trademark. manufacturer, or otherwise does not necessarily constitute or imply its errudorsement. recommendation, or favoring by the United States Government or any agesscy thereof. The views and opinions of authors expressed herein do not necessarily state or reflect those of the United Slates Governnent or any agency thereof.
\end{abstract}

\footnotetext{
tPermanent address: Associazione Euratom-Enea sulla Fusione, Ceatro Ricerche
} Energia Frascata, Go044 Frascati, Rome Italy 


\section{INTRODUCTION}

rrapped particle effects on plasma stability have been a subject of much therretical study, but trapped particle Instabilities have thus fiar fajled to play an important role in the description of large scale plasma behavior. Recently, however, instabilities have been observed, associated with neltral beam injection heating in tokamaks, which appear to be partly dut to trapped particle effects. ${ }^{-4}$ In previous work it was shown that near marginal stability for the internal kisk mode, high energy trapped particles introduce an additional destabilizing influence, producing a new branch to the internal kink mode dispersion relation, with a low threshold in the high energy trapped particle $\beta$, typically below one percent. Here $B$ is the ratio between particle and magnetic pressures. This trapped particle branch has a frequency near the totoidal precession frequency of the trapped particies and a growth rate of the same magnitude. For a model slowing-down bear particle distribution with a single value of $a=\mu / E$, with $\mu$ the magnetic moment and $E$ the energy, the Aispersion relation was calculated explicitly.

Further, it was shown ${ }^{6}$ that a mode with low toroidal and poloidal mode numbers, rotating iocoidally in resonance with the trapped particies, is capable of rapidly ejecting them from the plasma. These two features provide the basis for a model of the full fishbone instability cycle. Simulations of this cycle using a Hamiltonian guiding center orbit code wikl be reported in a Euture publication. In this work we examine the dispersion relat on for more general particle distributions. For simulation of the full cycle the particle dynamics under the action of the mode is followed through a Monte Carlo discrete particle representation. In order to study the coupled system of particle distribution and mode, we adopt a discrete particle zepresentation of the dispersion relation. The dispersion relation is first discussed 
qualitatively using the Nyquist method and then investigated numerically, and we examine the dependence on hot particle distribution, background plasma beta, and current profile. In Sec. II the dispersion relation for the internal kink mode in a plasma with a hot particle component is derived. In Sec. III the general properties of the solution are discussed, using a Nyquist analysis, ard it is shown that they are independent of the analytical form of the distribution function. In Sec. IV the numerical solution of the dispersion relation is discussed as a function of the parameters describing the relutively cold background plasma and the hot particles. Significance for plasma heating is discussed in sec. v.

\section{DISPERSION RELATION}

The plasma is considered to consist of two components, a relatively cold MHD part, and a hot trapped particle com, sonent, treated with a gyrokinetic description. $:^{3}$ In the grrokinetic description each particle is characterized by an energy $E$, magnetic moment $\mu$, and minor radius $r$. Finite banana width corrections to the particle dynamias and path integrals are ignored. The magnetic field is approximated by a low B circular equilibrium field, $B=(1-$ $I \cos \theta$ ), where $B$ is rormalized to its value on axis, and $r$ to the major radius. The particle energy is

$$
E=\frac{1}{2} v_{\|}^{2}+\mu B+\infty
$$

where $\phi$ is the potential energy associated with an electric field, and we have taken the particle mass to be unity. The volume in velocity space then becomes 


$$
d^{3} v=\frac{2 \pi v_{\perp} d v_{1} d v_{\|}}{(2 E)^{1 / 2}}=\sum_{0} \frac{2 \pi \operatorname{EdEA}_{\mu}}{(2 E)^{1 / 2}\left[1-c^{B}\right]^{1 / 2}}
$$

where $\alpha=\operatorname{sign}\left(\psi_{4}\right)$, and $\alpha=\mu / E$. The volume in the toroidal coocdinates $\phi r$, $\theta$ is

$$
d^{3} x=x d \phi r d \theta d r
$$

with $x=R+r \cos \theta$.

We begin with the expressions for the ideal MHD and the kinetic energy, given in Ref. 5. The ideal MHO energy is well known. 9 In deriving the expression for the kinetic energy of - ie trapped particle component, several physical assumptions weze made. The calculation is done only to lowest order in the inverse aspect ratio $\varepsilon=a / R$. The relative temperatures of the cold and hot components is $T_{c} / T_{h} \sim O\left(E^{2}\right)$. The poioidal beta values of the two components are taken to be of order $\beta_{p c} \sim 0(1)$ and $\beta_{p h} \sim 0(\varepsilon)$. Tnus $n_{h} / n_{c} \sim$ $U\left(\varepsilon^{3}\right)$ and overall charge neutrailty may be assumed. For mode frequencies small compared to hot particle transit frequencies and bounce frequencies, the perturbed trapped and untrapped distributions can be found. A variational dispersion functionel is then fourd of the form

$$
D(\vec{\xi})=\delta W_{M H D}+\delta W_{k}+\delta I
$$

where $\delta W_{M H D}$ and $\delta W_{k}$ refer to $M H D$ and kinetic contributions and the inertial term is

$$
\delta I=-\frac{1}{2} \omega^{2} \int d^{3} \times \rho_{m}|\xi|^{2}
$$


There is an inertial singular layer of a width $\Delta=\left(w_{f} / w_{A}\right)$ at $q\left(r_{s}\right)=1$, and only in this layer is the contrlbution of $\delta$ I significant, due to the singular nature of the plasma flow there. Here $\omega_{A}=v_{A} /\left(3^{1 / 2} \hat{R}\right)$ and $\hat{s}=r_{s} q_{S}^{\prime}$ is the local shear. Let $D=D_{e}+D_{5}$, where $L_{e}$ and $D_{s}$ are the contributions from outside and inside the singular layer, respectively. For the case of circular cross sections we have, outside the singular layer, $\vec{\xi}$ given by Bussac et $\underline{\text { al. }} .9$ We then obtain $D_{e}=\delta^{(2)}$ W $_{M H D}\left(\vec{\xi}^{e}\right)+\delta^{(2)} w_{k}\left(\vec{\xi}^{e}\right)+o\left(\varepsilon^{4}\right)$. These MHD and kinetic contributions to D are given by

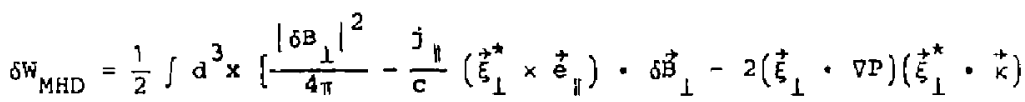

$$
\begin{aligned}
& \left.+\frac{\mathrm{B}^{2}}{4 \pi}\left|\vec{f} \cdot \vec{\xi}_{\perp}+2 \vec{\xi}_{\perp} \cdot \vec{\kappa}\right|^{2}+\gamma_{\odot}^{\mathrm{P}}|\vec{\gamma} \cdot \vec{\xi}|^{2}\right], \\
& \delta w_{k}=2^{9 / 2} \pi^{3} \pi_{h} \int \operatorname{RBr} d r \int d u \int_{0}^{\infty} d E E^{3 / 2} k_{b} \frac{\vec{J}^{*} \mathrm{~g}^{\bar{J}}}{\left(\omega_{d^{-}}-\omega\right)}
\end{aligned}
$$

The function $\mathrm{K}_{b^{+}}$related to the particle bounce period, is given by

$$
\mathrm{K}_{\mathrm{b}}=\frac{1}{\pi} \int_{0}^{\theta_{\mathrm{b}}} \frac{\mathrm{d} \theta}{[1-\mu E / E\}^{1 / 2}}=\left(\frac{2}{\mathrm{rq}}\right)^{1 / 2} \frac{\mathrm{k}\left(\mathrm{k}^{2}\right)}{\pi}
$$

where $\theta_{b}$ is the bounce angle, $B\left(r, \theta_{b}\right)=E / \mu$ In addition, we will need the bounce averaged quantity

$$
K_{2}=\frac{1}{\pi} \int_{0}^{\theta_{b}} \frac{d 0 \cos \theta}{\{1-\mu B / E\}^{1 / 2}}=\left(\frac{2}{r_{\alpha}}\right)^{1 / 2} \frac{\left[2 \mathrm{E}\left(\mathrm{K}^{2}\right)-\mathrm{K}\left(\mathrm{k}^{2}\right)\right]}{\pi},
$$

where $E\left(k^{2}\right), K\left(k^{2}\right)$ are complete elliptic integrals with $k^{2}=(E / \mu+r-1) /(2 r)$ The bounce averaged toroidal precession frequency is ${ }^{10}$ 


$$
\omega_{d}=\frac{\left[2 E\left(k^{2}\right)-k\left(k^{2}\right)\right] E q}{m r k\left(k^{2}\right) \omega_{0}^{R}}=\frac{x_{2}^{E q}}{k_{b} m \omega_{0}^{R}}
$$

and $\omega_{0}$ is the eyclotron frequency. The integral Eq. (7) defines $\delta . t_{k}$ in the upper half w plane, with definition in the lower half plane being given by analytic continuation, similar to the definition of the plasma dispersion function. 11 The function $J=(\alpha B / 2) \stackrel{+}{\nabla} \cdot \vec{\xi}_{1}-[1-(3 \alpha B / 2)] \vec{\xi}_{\perp} \cdot \vec{\kappa}$ with $\vec{\kappa}$ the field curvature, $\vec{k}=\partial \vec{e}_{\|} / \partial \ell, Q=\omega \partial F / \partial E-(\partial F / \partial r) /\left(\omega_{0} r\right)$, and $F$ is the hot particle distribution function.

Minimization of $\mathrm{D}\left(\vec{E}_{3}\right)$ to lowest order in $E$ involves only $\delta \mathrm{W}_{\mathrm{MHD}}$ tecause of the ordering, ant gives $\vec{\nabla} \cdot \vec{\xi}_{1}=-2 \vec{\xi}_{1} \cdot \vec{k}_{\text {. }}$ To evaluate $s w_{k}$, the lowest order expressinn for $\vec{\xi}$; sufficient, i.e., $\vec{\xi}_{1}=(1, i) \xi_{0}$ exp $[i(\phi-\theta)]$, for $0<r<$ $r_{s}$ and zero for $r>r_{s}$. Using this expression, we find the bounce averaged $J$ to he

$$
\vec{J}=\frac{k_{2}}{k_{b}} \frac{\xi_{0}}{2 R} \exp \left(i_{\phi}\right) .
$$

The inertial term $S I$ is found by solving the Euler equation for $\stackrel{+}{\xi}$ within the singular layer and matching it to the external solution. ${ }^{12}$ The internal kink mode is ristinct from other ideal MHD modes in that the singular nature of th: flow in tue singular layer makes the inertial term linear in $w$, converting the ideal dispersion relation from an equation for $\omega^{2}$ to an equation for $\omega$. We thus find

$$
D_{s}=\delta w_{M H D}^{(2)}\left(\vec{\xi}^{5}\right)+\delta I^{(2)}\left(\frac{\xi}{\xi}\right)+O\left(\varepsilon^{4}\right)=2 \pi R\left(\frac{B r}{2 R}\right)^{2}\left|\xi_{o}\right|^{2}\left(-\frac{i \omega}{\omega_{A}}\right)+O\left(\varepsilon^{4}\right) .
$$

Define the normalized distribution function through $E(r, \alpha, E)=2^{5 / 2} \mathrm{RE}^{1 / 2}$ 
$F / N_{p}$ where $N_{p}$ is the number of particles. Then $d^{3} N=N_{p} f K_{b}$ rdrdudE. We then find for the average trapped particle $B$ within $q=1, \beta=\left[\int d^{3} x\left(\theta_{\pi} P_{1}\right) / B^{2}\right] / V$ with $p_{\perp}=\int d^{3} \mathrm{vEF}$, the expression

$$
B=c_{\beta} \int r d r d a d E K_{b} E f
$$

with $c_{B}=\left(4 \pi^{2} \mathrm{~N}_{p}\right) /\left(\operatorname{RBr}{ }_{s}^{2}\right)$.

Substituting $\vec{\xi}_{1}$ and $F$ into $\delta W_{k}$, normalizing $\delta W$ through $\delta \hat{W}=2 R \delta \omega /\left(\pi^{2} B^{2} r_{s}{ }^{2} \xi_{0}{ }^{2}\right)$. dropping terms of bigher order in $\varepsilon$, and integrating by parts, we find

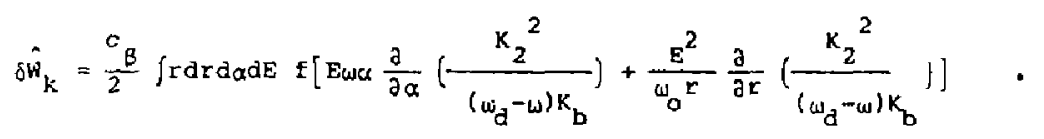

Part of the contribution of $\delta W_{M H D}$ is due to the hat component pressure $P_{1}$, coming from the term $-f d^{3} x\left(\xi_{\perp}, \nabla_{1}\right)\left(\xi_{1}^{*} \cdot x\right)$. Substituting $F_{1}$, we find this contribution to be

$$
\delta \tilde{W}_{M H D, h}=-\frac{C_{B}}{2} \int \mathrm{rdrdadEf} \frac{K_{2} E}{\mathrm{r}}
$$

Combining the MHD and kinetic parts, we then find for the dispersion relation

$$
0=D(\omega) \equiv-\frac{i \omega}{\omega_{A}}+\delta \hat{w}_{C}+\frac{C_{\beta} \omega}{2} \int r d r d \omega E E E\left(\frac{(A-B)}{\omega_{d}-\omega}-\frac{D_{\omega}}{\left(\omega_{d}-\omega\right)^{2}}\right)
$$

where

$$
A=\left[\frac{R K_{2}}{r}+R \frac{\mathrm{dK}_{2}}{\mathrm{dr}}+\alpha \frac{\mathrm{d}}{\mathrm{d} \alpha}\left(\frac{\mathrm{K}_{2}^{2}}{\mathrm{~K}_{\mathrm{b}}}\right)\right]
$$




$$
B=\left(k_{2} R \frac{d_{w_{d}}}{d r}+\alpha \frac{k_{2}^{2}}{k_{b}} \frac{d w_{d}}{d a}\right)\left(\frac{1}{w_{d}}\right)
$$

Energy dependence in Eq. (16) appears through $f(x, \alpha, E)$ and $\omega_{d}=g(x, a) E$. Evaluating this expression at threshold, $\omega=\omega_{r}+i \gamma, \gamma+0, \gamma>0$, we find from the imaginary part $1 / \omega_{A}=\pi C_{\beta 0} R\left(\omega_{Y}\right) / 2$, giving for the threshold $\beta$,

$$
\beta_{C}=\frac{2}{\pi \omega_{A} R\left(\omega_{r}\right)} \int r d r d \alpha d E K_{b} E E
$$

with the cesidue given by

$$
R\left(\omega_{r}\right)=\int \operatorname{rid} d_{\alpha}\left[\frac{(A-B) f E}{q}-\frac{\omega_{r}}{g^{2}} \frac{d}{d E}(E E)\right] \frac{\omega_{d}}{\prod_{d} T}
$$

evaluated at $E=\omega_{\mathbf{r}} / g$. The threshold frequency $\omega_{\boldsymbol{r}}$ is determined by the reat part of $\mathrm{Er}$. (16), which containg $\delta \hat{H}_{c^{\prime}} \mathbf{i} \cdot \mathrm{e}$, it is given by solving

$$
\left.0=\delta \hat{w}_{c}+\frac{C_{30}}{2} j \operatorname{rdrdudE} f E_{j i}, \frac{(A-B)}{1-\jmath 2}-\frac{B_{32}}{(1-3\})^{2}}\right]
$$

with $: \Omega=w_{r} / w_{d}+i \delta, \delta+0, ?: 0$. Thus $w_{k}$ is given by a weighted integral of $w_{d}$, and in general the threshold $\beta$ value depends on $\hat{w}_{c}$ through $\omega_{r}$. In the lower half plane $2 \pi i k$ must be added to the integral in $r_{g} .(16)$, with $R$ evaluated at the complex frequency $\omega$.

The solution to Eq. (21) is clearly dependent on the particle distribution. However, we have found that the general features of the solution, and to a large degree its quantitative properties, are independent of the detailed form of the distribution $f$. The cases we have examined numerically include model distributions for neutral beam injection, ion cyclotron heating, and fusion product a-particles. In addition, we have used 
particle distrinutions ${ }^{i 3}$ generated by codes which simulate realistic neutral beam injection, including slow down and scattering, as well as the modification of these distributions produced by the fishbone mode. The essential propertieg of the solutions are Identicai for all these cases. We illustrate the behavior of the solution using two examples, model distributions for neytral beam injection ard ion cyclotron heating. In the case of neutral beam injection, the inltial pitch of a particle is determined by the field at the point of ionization and the infection angle. In the large aspect ratio approximation this value is approximately the same for aj.l beam particles, $v_{\|} / v=\lambda_{0}$, which corresponds to a fixed value of $\mu / E=\left(1-\lambda_{0}{ }^{2}\right) / B$. Slowing down of the bean. through electron drag, described by aE/dt = - vE then gives a distribution of the form $\tilde{F}(\mathrm{E}) \sim 1 / \mathrm{E}, \mathrm{E}<\mathrm{E}_{\max }$.

In the case of ion cyclotron heating the banana tips of a rescnant particle mugt remain on the resonant surface, $R=R_{I}$, which implies a unigue value of $a=R_{L} / R_{0}$ for heating. In this case, we assume that the energy Aistribution assumes the form of a Maxwellian, $f(E) \sim e^{-E / E_{m}}$.

\section{GENERAL PROPERTIES OF THE SOLUTION}

Wrile the exact solution of Eq. (16: requires the fully numerical evaluation of $\delta W_{k}$, examined in the next section, we first discuss the general properties of the solution. Our aim is to derive some general criteria fur the occurrence of the instability as well as to check the dependence on the particular form of the cistribution function used. We use a Nyquist analysis. which is probably the moe suitable anong the anatycal approaches, consider a path in the complex w plane which runs between -o and $+\infty$ jugt above the real axis and then closes along a half circle in the upper half plane, shown in Fig. 1. The number of times the equivalent path in the complex plane 
encircles the origin equals the number of unstable roots of the equation $D(\omega)$ $=0$. In order to obtain a simple expression for $D(\omega)$ we evaluate the contribution of the trapped particleg retaining only the average effect of the curva wure and so we assume that the argunents of the elliptic integrals are not functions of $r$ and $a$, but can be replaced by some mean value. Under this approximation the radial dependence of the toroidal drift frequency is retained through the poloidal wave number only [the explicit $1 / x$ dependence in Eq. (10)]. In addition, we take a distribution tunction which is zero except for $\alpha=a_{0^{*}}$ Writing $f(r, \alpha, E) \propto n_{0}(r) E(E) \delta\left(\alpha-a_{0}\right)$ and uging Eq. (13), Eq. (16) can be written

$$
D(\omega)=-i \frac{\omega}{\omega_{A}}+\delta \hat{W}_{c}+\frac{3}{4 a} \frac{1}{K} \frac{B}{r_{s}} \frac{\omega}{\omega_{d s}}\left[\hat{A}-\hat{B}-\hat{B}_{\omega} \frac{\theta}{\partial \omega}\right] \int_{\eta}^{1} \operatorname{att}{ }^{1 / 2} H\left(t \frac{\omega}{\omega_{d s}}\right)
$$

where $t=r / r_{s}$

$$
\begin{aligned}
& \omega_{d s}=\frac{(2 E-K) E_{t} q}{r_{s} K \omega_{0}}, \\
& H(\xi) \equiv \int_{0}^{\infty} d E \frac{E E(E)}{E-\xi}, \\
& \hat{A}=\frac{1}{2}(2 E-K)\left[1-2 \frac{d}{d k^{2}} \frac{E}{K}\right]-\left[\frac{E}{K}+k^{2}-1\right] \frac{d}{d k^{2}}(2 E-K), \\
& \hat{B}=-(2 E-K)-2\left[\frac{E}{K}+k^{2}-1\right] K \frac{d}{d k^{2}} \frac{E}{K}, \\
& a=\int_{0}^{\infty} d E E E(E),
\end{aligned}
$$

and all elliptic integrals are independent of $r$. In the following we shall assume $n=\left|1 / r_{0}-1\right| R / r_{S} \ll 1$. In the expression for $4 f_{5}$, $E_{m}$ is a 
characteristic energy, equal to the temperature in the case of a maxwellian distribution function $f(E) \sim \exp \left(-E / E_{m}\right)$, and to the maximum energy in the case of a slowing down distribution function, $f(E) \sim 1 / E$.

A. Slowing-down beam population

For the beam case the function $H(\xi)$ reduces to

$$
H(\xi)=\ln \left(1-\frac{1}{\xi}\right)
$$

Carrying out the radial integration, the final expression for $D(w)$ becomes

$$
\begin{aligned}
D(\omega) & =-i \frac{\omega}{\omega_{A}}+\delta \hat{W}_{C}+\frac{1}{2} \frac{1}{\mathrm{~K}} \frac{\beta}{\Sigma_{S}}\left\{(\hat{A}-\hat{B}) \Omega\left[\ln \left(1-\frac{1}{\Omega}\right)\right]+\right. \\
& \left.+\left(\hat{A}+\frac{1}{2} \hat{A}\right)\left[\Omega^{-1 / 2} \ln \frac{1+\Omega^{1 / 2}}{1-\Omega^{1 / 2}}-2\right]\right\}
\end{aligned}
$$

where $\Omega=\omega / \omega_{d s^{*}}$ If the $(\hat{A}+\hat{B} / 2)$ term is neglected, $D(\omega)$ reduces to the expression studied in Ref. 5. The effect of the new term is negligible for $\omega<\omega_{\text {dg. }}$ It ig due to particles at small $r$ with $w_{d}>w_{\text {ds }}$. Due to the nonalalyticity of the function $H(\xi)$, we have to deform the path in the complex $\omega$ plane around the points $\omega=0$ and $\omega=\omega_{\mathrm{dg}}$, as shown in Fig. 1. Looking first at the Nyquist diagram and assuming ubs $>0$ (beam particles predominantly in the bad curvature region), it is seen that the imaginary part of $\mathrm{D}$ is given by 


$$
-w^{\prime} \omega_{\mathrm{A}}
$$

$\operatorname{ImD}=-\left(\omega / \omega_{A}\right)\left(1-\beta / \beta_{C}\right)$

$$
-\omega / \omega_{A}+\frac{\pi}{2} \frac{B}{r_{s}} \frac{1}{K}\left(\hat{A}+\frac{1}{2} \hat{B}\right)\left(\frac{\omega}{\omega_{d s}}\right)^{-1 / 2}
$$

If in $E q$. (25) the $\hat{A}+\hat{B} / 2$ term is neglected, then Im $D=0$ for $\omega E C_{1}$ if $B<H_{C}$ and for $\omega \in C_{2}$ if $\beta>B_{c}$, (see Fig. 1) where $\beta_{c}$ is defined by

$$
\hat{B}_{C}=\frac{2}{\pi} \frac{r_{s}}{R} \frac{\omega_{d s}}{\omega_{A}} \frac{K}{\hat{A}-\hat{B}}
$$

Taking into account the expression for Re $D$, we find that for instability either ${ }^{\hat{w}_{C}}<0$ and $\beta<\beta_{C^{\prime}}$ or simbly $\beta>\beta_{C}$. These two conditions refer to two different branches. Note that in both cases the path in the $D$ plane encircles the origin only once and so for a given value of $p$ and $\delta \hat{H}_{c}$ at most onc hranch of the dispersion relation can be unstable. The unstable branch is a MHD mode or a trapped particle $(T)$ mode deperding on the value of $\gamma$ at $\beta=0$. The Nyquist diagrams for $\beta\left\langle\beta_{C}\right.$ and $B>B_{C}$ are shown in Fig. 2. We can gain further insight into the behavior of the solution of Eq. (25) by examining the -hreshold frequency for $\beta=\beta_{c}$, which lies in the range $0<\omega<\omega_{\mathrm{ds}}$. To this aim $w=2 \equiv n$ use the simplified form of the real part of Eq. (25),

$$
:=\therefore=-\frac{1}{T} \frac{\partial}{\omega_{A}} \text { en }\left|1-\frac{w_{d s}}{\omega}\right| \text {. }
$$

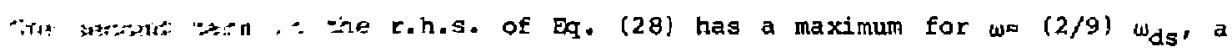

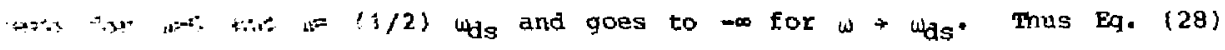

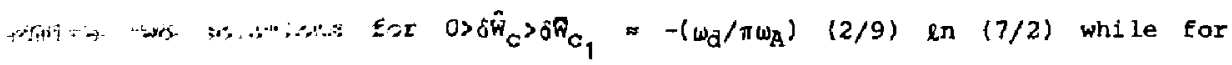


$\delta \hat{W}_{c}<\delta W_{c 1}$ no marginal modes are present. Linearizing Eq. (25) around the threshold solution we find for the growth rate

$$
Y=-\omega_{Y} \frac{\beta-\beta_{c}}{\beta_{c}} \frac{\pi(s)}{\omega_{d s}} \frac{1}{\ln \left(\omega_{\mathrm{d} \theta} / \omega_{Y}-1\right)-\omega_{r} /\left(\omega_{\mathrm{ds}}-\omega_{Y}\right)}
$$

where $\omega_{Y}$ is the solution of Eq. (28). From Eq. (29) it is possible to see that the $M F D$ mode is unstable for $B<\beta_{c}$ while the $T$ mode is unstable for $\beta>\beta_{C}$. For $\delta \hat{W}_{C}>0 \mathrm{Eq}$. $(2 \theta)$ admits only one solution in the range $0<\omega<\omega_{\mathrm{ds}}$ which corresponds to $T$ mode and for $\delta \hat{W}_{c}+\infty\left(\omega_{0}+4 d s\right)$ the growth rate becomes exponentially small, $Y \sim \exp \left(-\pi\left[\omega_{A} / 2 \omega_{d s}\right] d \hat{w}_{C}\right)$. If the second term in gq. (25) is taken into account, the behavlor of the solution of the dispersion relation changes only for $\omega s \omega_{d s}$. In particular, the only relevant effect is that the I mode is stable for

$$
B<\frac{\pi \hat{\beta}_{c} \delta \hat{W}_{C} \omega_{A}}{w_{d}(1+(2 \hat{A}+\hat{B}) /(\hat{A}-\hat{B})]}
$$

and $\beta \gg \beta_{C^{*}}$ In fact for smaller $\beta$, in the domain given by $E q .(30)$ and $\beta \geqslant$ $\beta_{c}$ the mode, while unstable, has an exponentially small growth rate, and thus in practice Eq. (30) can be regarded as defining a stable regime for all $\beta<$ $B_{C}$

в. Maxwellian distribution

In the case of a Maxwellian distribution function, $H(E)$ is related to the plasma dispersion function

$$
H(\xi)=\sqrt{\pi}\left[\frac{1}{2}+\xi+\xi^{3 / 2} \mathrm{z}\left(\xi^{1 / 2}\right)\right]
$$


and the usual analytic continuation for Im $\xi<0$ holds. Using the relation $Z^{\prime}(t)=-2[1+t z(t)]$, it easy to carry out the radial integration in Eq. (22). The expression for $D(\omega)$ is

$$
\begin{aligned}
& D(\omega)=-i \frac{\omega}{\omega_{A}}+\delta \hat{H}_{c}+\frac{1}{K} \frac{B R}{x_{S}}\left\{\left(\hat{A}+\frac{1}{2} \hat{B}\right) i d \pi s i^{-1 / 2}\right. \\
& -\left(\hat{A}+\frac{1}{2} \hat{B}\right) \quad u\left[1+\frac{4}{32}+u^{-3 / 2}\left(\Omega^{2}+2 n+2\right) z\left(u^{1 / 2}\right)\right] \\
& -B_{2}\left(\frac{l}{2}+s+12^{3 / 2} z\left(s^{1 / 2}\right)\right)
\end{aligned}
$$

Besides the $w=0$ solution, the real zeros of the imaginary part of $D(w)$ are given, after some algebra, by the roots of the following pquation

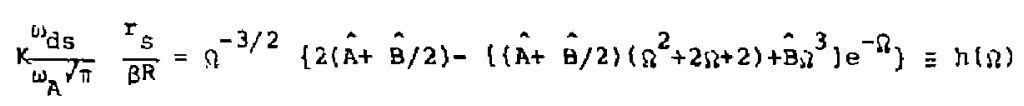

The r.h.s. of $\mathrm{FG} .(33)$ is positive and has a maximum for $\Omega=\Omega_{0} \sim 1$. So Eq. (33) admits solutions only for 3 larger than some minimum value $B_{m}$ given by

$$
B_{\mathrm{m}}=\frac{w_{\mathrm{ds}}}{w_{\mathrm{A}} \sqrt{\pi}} \frac{\mathrm{r}}{\mathrm{R}} \mathrm{h}^{-1}\left(s_{\mathrm{o}}\right)
$$

Using the asymptotic expansion of $h(\Omega)$, the two solutions for $B>B_{m}$ are

$$
s=\left[\frac{3 K \omega \omega^{r} s}{(\hat{A}-5 \hat{B} / 2)\left(\sqrt{ } \omega_{A} \beta R\right)}\right]^{2 / 3}
$$

and 


$$
w=\left[\frac{2(\hat{A}+\hat{B} / 2)\left(r \pi \omega_{A} \beta K\right)}{K \omega_{d s} Z_{s}}\right]^{2 / 3}
$$

The corresponding valucs of the real part of 0 are

$$
\begin{aligned}
& \operatorname{Re} D=\delta \hat{H}_{c}+1 /\left(3^{1 / 3} / \pi h^{1 / 3}\left(\Omega_{0}\right)\right) \frac{\omega_{\mathrm{ds}}}{\omega_{\mathrm{B}}}(\hat{\mathrm{A}}-\hat{\mathrm{B}}) /(\hat{\mathrm{A}}-5 \hat{\mathrm{B}} / 2)^{2 / 3}\left(\frac{B}{\beta_{\mathrm{m}}}\right)^{1 / 3} 3 \ll<1 \\
& \text { Re } D=\delta \hat{w}_{C}-\frac{3}{2 \sqrt{\pi}} \frac{\hat{A}}{h\left(\Omega_{0}\right)} \frac{\omega_{d s}}{\omega_{i}} \frac{B}{B_{m}}
\end{aligned}
$$

and the behavior of the Nyquist diagram can be understood in terms of these expressions.

For $\delta \hat{H}_{C}$ positive the MHD mode is stable, while the $T$ mode reaches marginal stability for $\beta \sim \beta_{m}\left(\omega_{A} / \omega_{d}\right)$ ô $\hat{w}_{C}$ at $\omega>>\omega_{A^{*}}$. For $\delta \hat{\omega}_{c}$ negative the MHD mode is unstable and the $T$ mode becomes unstable for $\beta \sim \beta_{m}\left(\omega_{A}\left|\hat{o} \hat{w}_{c}\right| / \omega_{d}\right)^{3}$. Above this value both the modes are unstable. The Nyquist diagrams for $B<E_{m}$ and $\beta>\beta_{m}$ are shown in Fig. (3),

We want to stress here that the behavior of the Nyquist diagram as well as the asymptotic behavior of the threshold value of $B$, show a more than qualitative agreement for the two cases studied, in spite of the different distribution functions. This is probably an indication that the occurrence of the instability is determined only by the value of $\beta$ and $\delta W_{c}$ and not by the details of the distributions. In general, a minimum threshold value of $B \sim\left(\omega_{d} / \omega_{A}\right) r_{S} / R$ exists for $\left|\delta \hat{w}_{C}\right|<\omega_{d} / \omega_{A} ;$ at larger and positive value of $s w_{C}$ the threshold value increases almost linearly $B \sim B \sim \beta_{m}\left(\omega_{A}\left|\delta \hat{\omega}_{c}\right| / \omega_{d}\right)$, while for larger and negative values of $\delta \hat{H}_{c}, i . e .$, under conditions of instability for tine MHD mode, the dependence of the threshold value of $\beta$ on $\delta \hat{w}_{C}$ is cubic $B \sim B_{1 i}\left(\omega_{A}\left|\delta \hat{H}_{c}\right| / \omega_{d}\right){ }^{3}$ 


\section{NUMERICAL SOLUTIONS}

Solutions of the dispersion relation, Eq. (16), were obtaiged numericaliy using an interactive scheme making use of the stokes plot ${ }^{14}$ in the complex w plane associated with $D(\omega)$. The particle distrj.bution was given by the discrete representation

$$
f=\frac{1}{N} \sum_{n=1}^{N} \delta\left(\alpha-\alpha_{n}\right) \delta\left(E-E_{n}\right) \delta\left(\frac{r^{2}}{2}-\frac{r_{n}^{2}}{2}\right)
$$

with $N$ the number of particles used in the simulation. A continuous oistribution $F(E, \bar{E}, r)$ wolld normally give rise to a cut in $D(w)$ along the real axis from zero to $\omega_{\mathrm{d}} \mathrm{m}^{\prime}$ the maximum arift frequency in the distribution. Because of the discrete particle representation of $F$ this cut is represented as $\exists$ sum of $N$ singularities. In addition, there are a large number of extraneous roots to $D(\omega)=0$, confined to a neighborhood of the real axis (within a distance given by the spacing between singularities). These Dawson, 15 or streaming solutions, make it impractical to solve the dispersion relation for $|\gamma| \lesssim \omega_{\mathrm{dm}} / \mathrm{N}$ where $\mathrm{N}$ is the number of particles in the Monte Carlo simulation. For small values of $y$ it is necessary to use an analytio expression obtained by a first order solution of the dispersion relation around the threshold.

The particle aistributions were generated using Monte Carlo technigues and random number sequences available on the CRAY 1 . The details of the method are illustrated for the first case considered.

A. Slowing-down population

We first consider the slowing-down population used in Ref. 5 ,

$$
F(E, \mu, r)=\frac{c(r)}{E^{3 / 2}} \delta\left(\frac{\mu}{E}-\alpha\right)
$$


This approximates the distribution found in a neutral beam heated plasma during beam injection. The number of particles in an element of phase space is $\mathrm{Fd}^{3} \mathrm{vd}{ }^{3} x$, giving the number in energy range dE proportional to $1 / \mathrm{E}$. Writing $\operatorname{dn} / \mathrm{dE}=(\mathrm{dn} / \mathrm{dx})(\mathrm{dx} / \mathrm{dE})=\mathrm{a} / \mathrm{E}$ and using a randomly generated uniform distribution in $x$ in the internal $\left(0, x_{\max }\right)$, i.e., $(d n / d x)=$ const, the correct enerqy distribution is generated through

$$
E=E_{\max } \exp \left(x-x_{\max }\right)
$$

with range $E_{\max } e x p\left(-x_{\max }\right)<E<E_{\text {max }}$ In addition, the particles are chosen to be Aistributed usiformly in volume, i.e., $r_{n} \sim(n / N)^{1 / 2} r_{s}$.

$$
\text { Using } f(x, \alpha, E)=a / E \text {, we find from Eqs. (19) and (20) for the critical }
$$
beta,

$$
\langle\beta\rangle_{\text {crit }}=\frac{2\left\langle K_{b} E\right\rangle\left(E_{\max }-E_{\min }\right)}{\pi \omega_{A}\left\langle(A-B) E^{2} / \omega_{d}\right\rangle}
$$

where the brackets indicate averages over the distribution, i.e., $\langle H\rangle=\int$ rarda defH. If the dependence of $u_{d}$ on $r$ and $\alpha_{\text {, }}$ and the dependence of $k_{b}$ on $r$ is neglerted, we find $B=0$ and

$$
\langle\beta\rangle_{\text {crit }}=\frac{2 \omega_{d m}}{\pi \omega_{A}} \frac{k_{b}}{\langle A\rangle}
$$

in agreemert with Ref. 5. These approximations typically give 〈B〉crit too large py a factor of two. However, as shown in Sec. III, the fact that this threshold value is independent of $\delta \hat{H}_{c}$ is misleading, because for values of $\delta \hat{w}_{\mathrm{c}}$ $>\omega_{d} / \omega_{A}$ and $G \geq \beta_{c}\left(\omega_{A}\left|\delta \hat{w}_{c}\right| / \omega_{d}\right)$, the growth is exponentialiy small. Thus the threshold behavior of the neutral beam and ion cyclotron cases can be regarded as essentially the same. 
Perturbing $\beta$ and $w$ about the threshold values, linear expressions for the

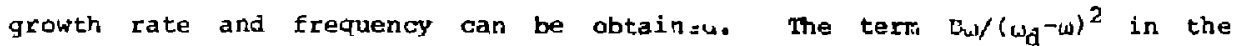
dispersion relation is easily shown to be negligible. We then find

$$
\begin{aligned}
& \frac{Y}{w_{r}}=\frac{\left(B-B_{C}\right)}{B_{c}} \frac{S_{1}}{\left(S_{3}-S_{2}\right)} \\
& \frac{\delta w_{E}}{w_{r}}=\frac{\left(3-B_{C}\right)}{B_{c}} \frac{S_{2}}{\left(S_{3}-S_{2}\right)}
\end{aligned}
$$

where the numerical factors $s_{1}, s_{2}, s_{3}$ are given by

$$
\begin{aligned}
& s_{1}=\left\langle E^{2} \frac{(A-B)}{\omega_{B}}\right\rangle \\
& s_{2}=\left\langle\frac{(A-B) E^{2} 2 n}{\omega_{d}}\left|\frac{E_{\max }-\frac{\omega_{I} E}{\omega_{d}}}{E_{\min }-\frac{\omega_{I} E}{\omega_{d}}}\right|\right\rangle
\end{aligned}
$$

and $S_{3}=\left\langle\frac{\omega_{r}(A-B) E\left(E_{\max }-E_{\min }\right)}{\left[\omega_{r}-\omega_{1} \frac{\left.E_{\min }\right)}{E} \frac{\left(\omega_{d} E_{\max }\right.}{E}-\omega_{r}\right)}\right\rangle$

From Fq. (21) we then have that for 3 a $B_{C}$ the growth rate, aside from a numerical factor, is given iy $\langle 4\rangle^{\rangle}$.

Shown in Figs. A through 6 are the results of a numerical investigation of the dispersion relation for the slowing-down distribution, with $a=0.99$, $w_{A}=3.8 \times 10^{6} \mathrm{sec}^{-1}$, and various $\delta \hat{w}_{c}$. We have used the equilibrium of Ref. 5 , with the $q=1$ surface located at $I_{s}=a / 2$, and $B=10 \mathrm{kG}$. We considered a $50 \mathrm{kev}$ deuterium ion injection. For these parameters $\omega_{\mathrm{dm}}=1.7 \times 10^{5} \mathrm{sec}-1$ and the average $w$ lue $\left\langle\omega_{d}\right\rangle=5 \times 10^{4} \sec ^{-1}$, In Fig. 4 are shown the MHD (M) 
and trapped particle ( $T$ ) branches of the dispersion relation, as a function of $B$, for various values of $\hat{s}_{c}$. The subscripts indicate the values of $\hat{c}_{c}$ in mu:ltiples of $2.5 \times 10^{-3}$, i.e., the curve $T_{ \pm n}$ has $\delta \hat{H}_{c}= \pm 2.5 \mathrm{n} \times 10^{-3}$. The trapped particle branch is located at $Y=-\infty$ for $B=0$, and appears in the lower half plane as 3 approaches the threshold value $\hat{f}_{c}$, which is independent of $\hat{\delta} \hat{w}_{c}$ for this distribution. Figures 5 and 6 give the dependence of $;$ and $w_{r}$ on $B$ for the same curves shown in Fig. 4.

The MHD branch, located at $Y=-\omega_{A} \delta \hat{W}_{C}$ and $\omega_{r}=0$ for $B=0$, is stabilized by the liapped particles for $\delta \hat{W}_{C}>0$. For $\delta \hat{w}_{C}<0$ the mode takes on a positive frequency as $B$ increases, but is stabilized as long as $y<\left\langle w_{d}\right\rangle$. As ofi. beromes more negative, and the MHD and trapped particle branches coalesce. As observed in Sec. III, at most one branch is unstable for given values of $\delta \hat{w}_{c}$ and $\beta$. For sufficiently negative $\delta \hat{w}_{C}$ this is the MHD branch, as displayed in Fig. 5 by the curves $M_{-2}$ and $T_{-2}$.

In Fig. 7 is shown the dependence of $y$ on $\delta \hat{w}_{C}$ for various alues of $\beta$, given by $B=n \times 10^{-3}$ for curve $r_{n}$. The trapped particle branch shows essentially exponential behavior, $Y \sim \exp \left(-a d \hat{\omega}_{C}\right)$ in agreenent wich the analysis of sec. III. In Fig. 8 is shown the frequency for the curves of Fig. 7.

B. Maxullian distribution

The second particle distribution we consider is a Maxwellian distribu'.ton function with a single value of $\mu / E$

$$
F(E, j, r)=n(r) e^{-E / T_{h}} \delta\left(\frac{\mu}{E}-\alpha\right)
$$


This distribution is a good approximation to the hydrogen distribution function found during the minority species ion cyclotron heating expeijments. The value of $n$ is typically of the order $10^{-2}$ times the average density and the value of the temperature ranges between 30 and $100 \mathrm{keV}$ depending on the injected power. Both these quantities can be assumed constant inside the $q=1$ surface. The value of $\alpha$ is obtained by requiring that the tips of the trapped particle banana orbits lie in the resonant layer. If $n_{\tau}$ is the distance of the layer from the axis of the torus, this condition can be written

$$
u=R_{I} / R \text {. }
$$

We have used the Monte Carlo techniques, as in the slow ciown case, to investigate the stability of this distribution function. The randon variable $x$ is related to the energy by the equation

$$
x(E)=\frac{\operatorname{erf} E^{1 / 2}-\operatorname{erf} E_{1}^{1 / 2}+(2 / \sqrt{ })\left[E_{1}^{1 / 2} e^{E_{1}}-E^{1 / 2} e^{-E}\right]}{\operatorname{erf} E_{2}^{1 / 2}-\operatorname{erf} E_{1}^{1 / 2}+(2 / \sqrt{ })\left[E_{1}^{1 / 2} E^{-E_{1}}+E_{2}^{1 / 2} E^{-E_{2}}\right]}
$$

where $\mathbf{E}$ is normalized to the temperature, $0<x<\uparrow$, and $E_{1}<E<E_{2}$. $A$ gualitative agreement of the behavior of the dispersion relation with the slow down case has been found.

A plot of the stable and uastable Iegions in the $B$, $\delta \hat{\mathrm{c}}$ plane is given ia Fig, 9 for the same equilibrium parameters and various positions of the resonant layer. Note that the range of $\delta \hat{w}_{c}$ covered is considerably larger than that given by the curves in Figs. 4 and 5, which, although describing the bedm injection case, show the same behavior. The dependence of the threshold 
beta on $a_{0}$ is easily interpreted physically and has important consequences for plasma heating. Large $\alpha_{0}$ corresponds to $v_{\perp} \gg v_{\|}$, i.e., near perpendicular beam injection and ion cyclotron heating in the deeply trapped, bad curvature region, where $\omega_{d}$ is largest. Not only is the threshold lower and the range of unstable $\delta \hat{w}_{c}$ larger when the particles are deeply trapped, but the particle ejection induced by the mode is more efficient. ${ }^{6}$ Thus experiments producing fewer deeply trapped particles should observe less frequent and less violent fishbone oscillations.

c. Fusion alpha particles

Particle distributions simulating fusion alpha particles were also generated using Monte Carlo techniques. The distribution was assumed uniform in pitch $\lambda$, and in initial location, $r$ and $\theta$. The distribution in $u=\mu / E$ is then given by $a=\left(1-\lambda^{2}\right) / B(r, \theta)$. A slowing-down distribution was assumed for the energy. Plasma parameters corresponding to a fusion reactor were used. This model profuces threshold values of $\beta$ of a fraction of a percent for $\delta \hat{w}_{c}=0$. Whis is approximately the value of $B$ due to Eusion alpha particles expected in a steady-state reactor. ${ }^{16}$ whether or not fusion a paricles will induce fisthone oscillations will thus depend on details such as th plasma $\beta$ obtained and the threshold for the internal kink mode, which depends also on plasma shaping and device parameters.

\section{v. CONCLUSION}

The destabilization of the internal kink mode by trapped high energy particles has been investigated for a number of distribution functions. The mode threshold and growth rate was found to be fairly independent of the form of the distribution function. The threshold trapped particle beta, $\beta_{c}$ has a 
minimum for $\delta \hat{\omega}_{c}=0$ and increases linearly in $\delta \hat{w}_{c}$ for $\delta \hat{w}_{c}>0$. For typical fusion experiment parameters the minimum $B_{c}$ is a fraction of a percent. The range of $\delta \hat{w}_{C}$ in which the mode is unstahle for a given $\beta$ decreases as the particle population becomes less deeply trapped. These results indicate twc effective mcans of avoiding the instability. plasma deformation can raise tha threshold of the internal kink, effectively increasing $\delta \hat{\psi}_{C}$ for a fixed particle distribution, and the heating mechanism can be modified to prosuce a popllation which is less deeply trapped. Fishbone oscillations induced by fusion alpha particles are a possibility, but their occurrence will depend on the details of the reacting plasma.

Other MHD modes, besides the internal kink, must be regarded as candidates for fishbone-like instabi ties. In particular, there is frequently observed a high frequency precursor to the fishbone oscillation, which has been tentatively identified as a localized interchange mode ${ }^{17}$ or ballooning modes ${ }^{18}$ excited by energetic tiapped particles. The attainment of higher beta plasmas will undoubtedly reveal other, related, instabilities.

\section{ACKNOWLEDGMENTS}

This work was supportei: by U.S. Department of Energy Contract No. DE$\mathrm{ACO} 2-76-\mathrm{CHO}-3 \mathrm{O} 73$. 


\section{REFERENCES}

${ }^{\top} \mathrm{k}$. MaGuire et aí., Phys. Rev. Lett. 50, 891 (1983).

$2_{J}$. L. Dunlap et al. Phys. Rev. Lett. 46,538 (1982).

${ }^{3}$ D.o. Overskei et al.. Proceedings of the Fourth International Symposium on Heating in Toroidal Plagmas, Rome, 1984.

45. Yamamoto et al. Nucl. Fusian 21, 993 (1981)

5L. Chen, R. B. White, and M. N. Roserbluth, Phys, Rev. Lett. 52, 1122

$(1984:$

${ }^{6}$. 8. White et al., Phys. Fluids 26, 2958 (1983),

7T. M. Antonson, Jr. and B. Lane, Phys. Fluids 23, 2205 (1980).

Bp. J. Catto, W. M. 'Tang, and D. E. Baldwin, Plasma Phys. 23, $639(1981)$.

9 M. N. Bussac, R. Pellat, D. Edery, and J, L. Soule, Phys. Rev. Lett. 35 , $1638(1975)$.

10 B. B. Karomtsev, in Plasma Phy;ics and the Problem of Controlled

Thermonuclear Reactions (Pergamon Press, New York, 1959), Vol. II , p. 340.

${ }^{11}$ B. D. Fried and S. D. C, :e, The Flasma Digpersion Function (Academic Pross Inc., New York, 1961).

12 M. N. Rosenbluth, R. Y. Jagazian, and P. H. Rutherford, Phys. Fluıds 16. $1894(1973)$.

${ }^{13}$ R. J. Hawryluk et a 1., in Physics of plasmas close to Thermonuclear Conditions (Comission of the European Comminities. Brussels, 1990) vol. 1.

14 R. B. White, J. Comput. Phys. 3i, 409 (1979).

$15 \mathrm{~J}$. Dawson, phys. Rey. 118, 3B1 (1960).

16D. Jassby (private communication).

17J. Manickam, K. McGuire, and A. E. Miller, presented at the Sherwood Fusion Theory Conference, Incline Village, Nevada (1984).

${ }^{18} \mathrm{~J}$. Weiland and I. Chen, presented at the sherwood Fusion Theory Conference, Incline village, Nevada (1984). 


\section{FIGURE CAPTIONS}

FIG, 1 The complex $w$ plane, showing the closed curve uged in the Nyquist analysis.

FIG. 2 The complex $D(\omega)$ plane for the slowing-down dictribution, showing the Nyquist plots for $\delta \hat{H}_{C}=5 \times 10^{-3}, \omega_{0}=1.05$, and $\beta\left\langle\beta_{C}, \beta\right\rangle \beta_{C}$.

Fig. 3. The complex $\mathrm{n}(\omega)$ plane, for the Maxwellian distribution, $\delta \hat{H}_{c}=0$, $C_{0}=1.05$, and $\beta<B_{C}, B>G_{C}$.

FIG. 4. Trapped particle ( $T$ ) and MHD (M) branches of the dispersion relation for tile slowing-down distribution, for various vajues of ôn "given by $\delta \hat{W}_{c}= \pm 2.5 n \times 10^{-3}$ for curve $T_{ \pm n}$.

FIG. 5. The growth rates $\gamma$ of the curves of Fig. $i$ as functions of $\beta$.

FIG. 6. Frequencies $w_{r}$ of the curves of Fig. 1 as functions of $\beta$.

FIG. 7. Growth rate as a function of ow for various values of $B$.

FIG. 8. The Erequency as a function of $\delta \hat{W}_{c}$ for the curves of Fig. 7.

FIG. 9. Critical beta, $\beta_{C}$ vs $\delta \hat{w}_{c}$ for a Maxwellian distribution, for two values of $\alpha_{0}$. 
25

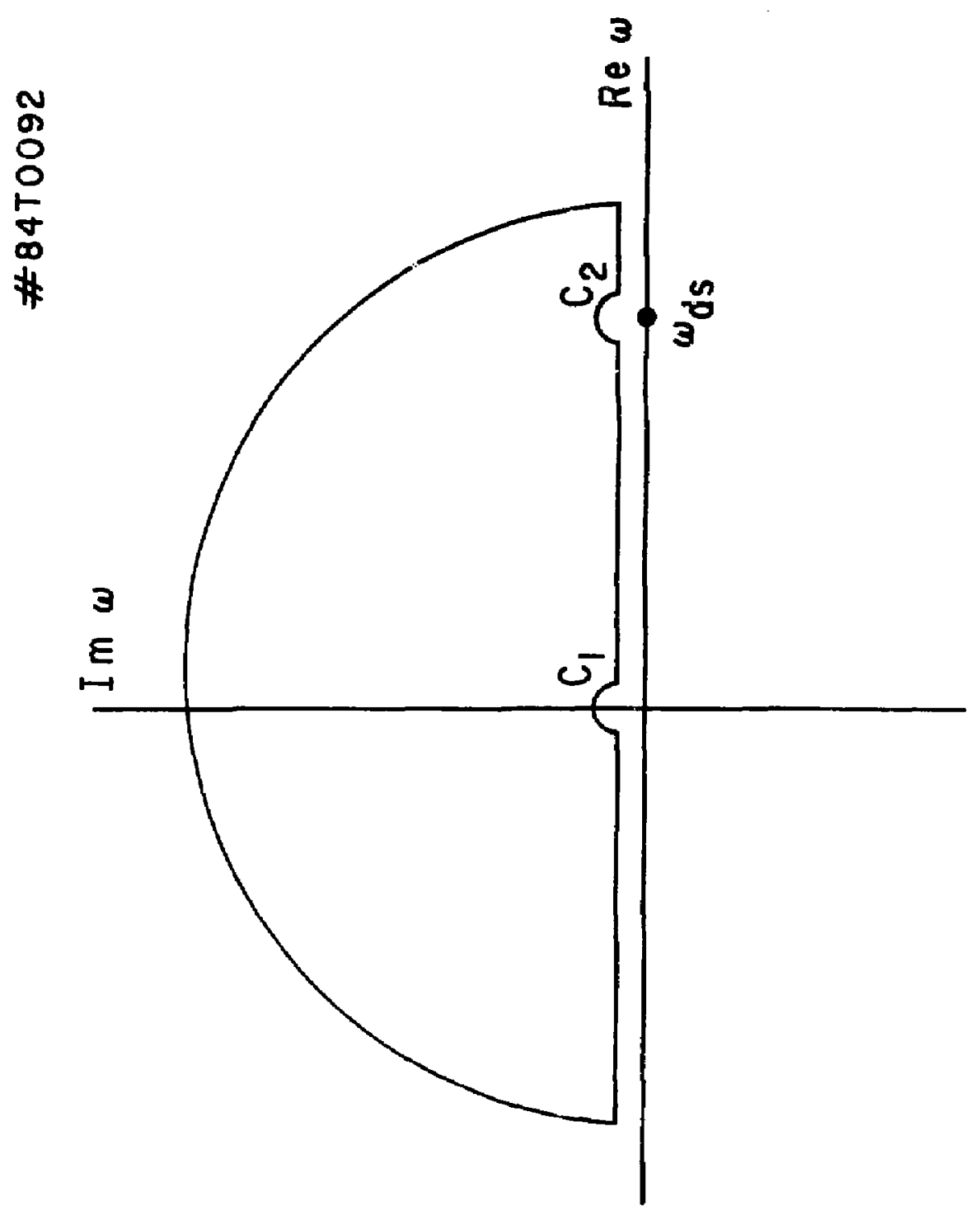


\#84 T0094

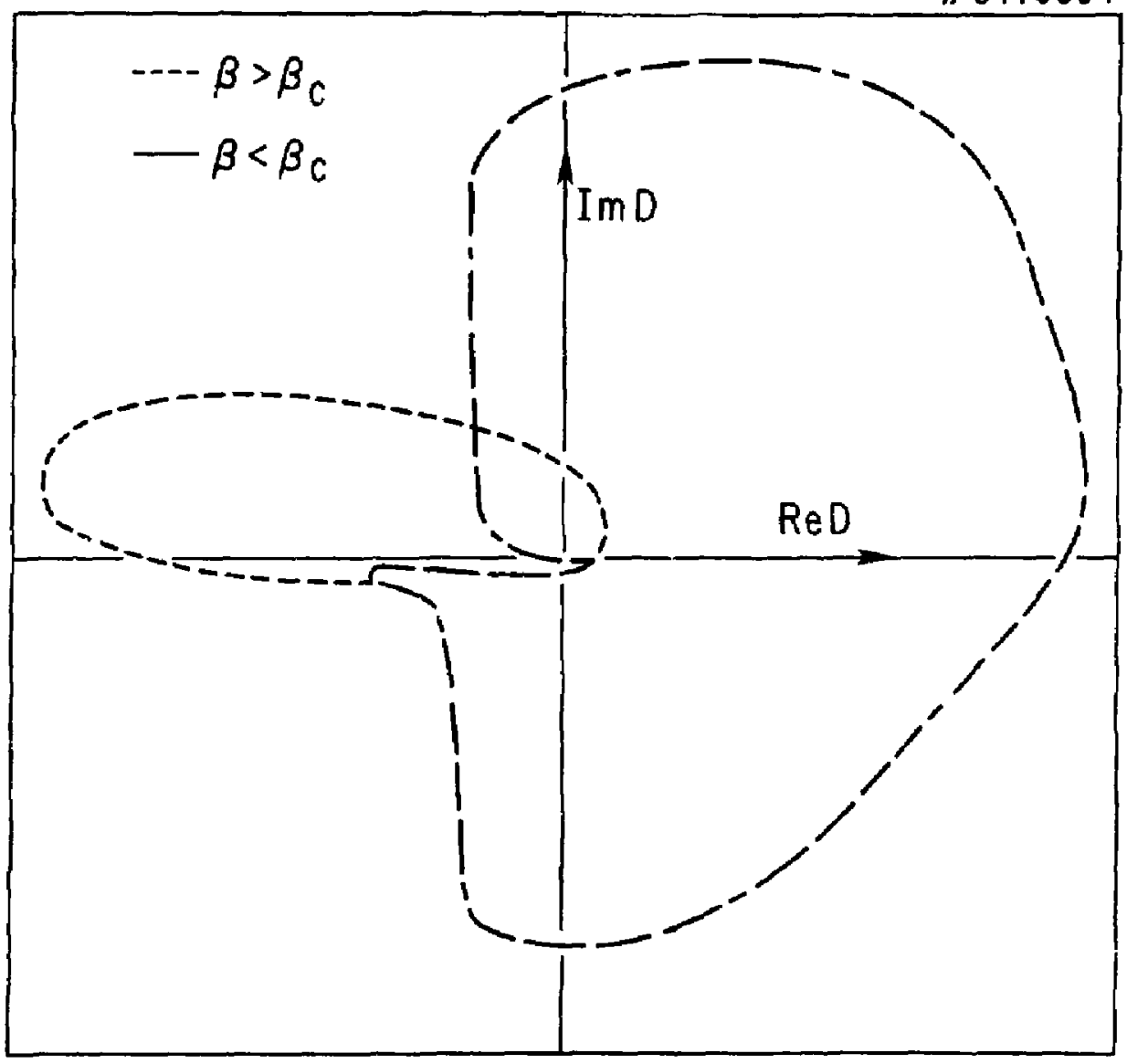

Fig. 2 


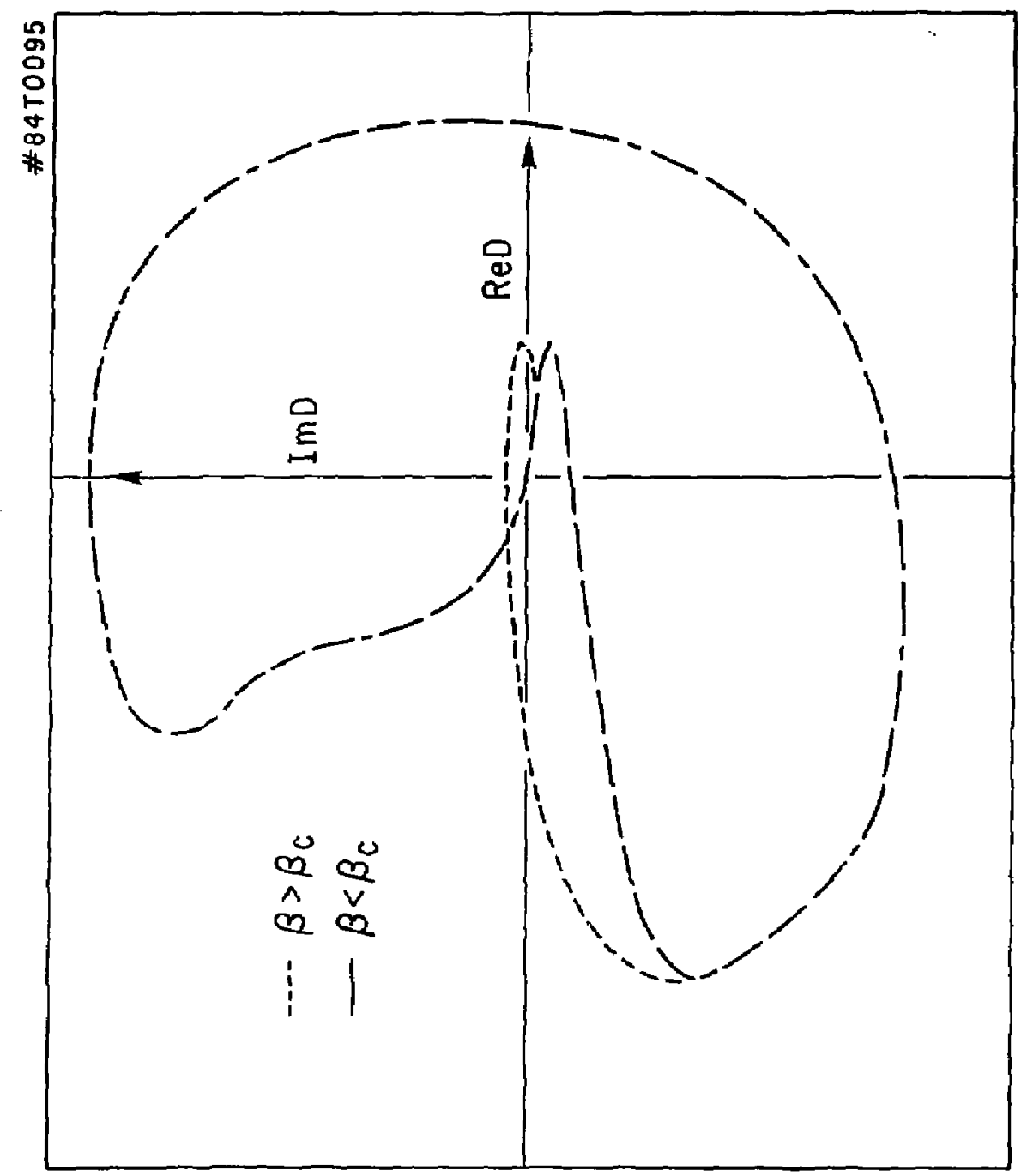

m 


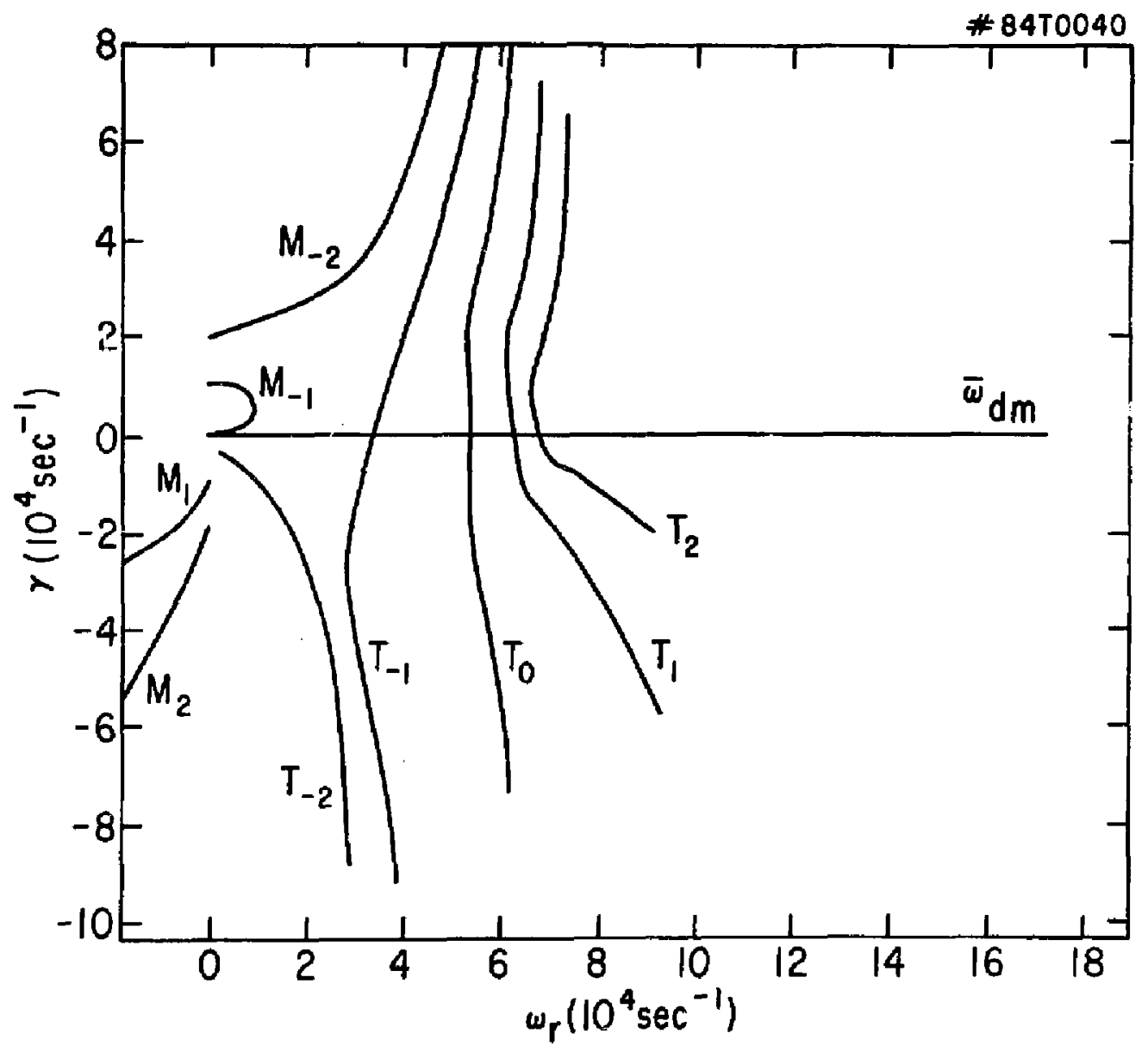

Fig. 4 


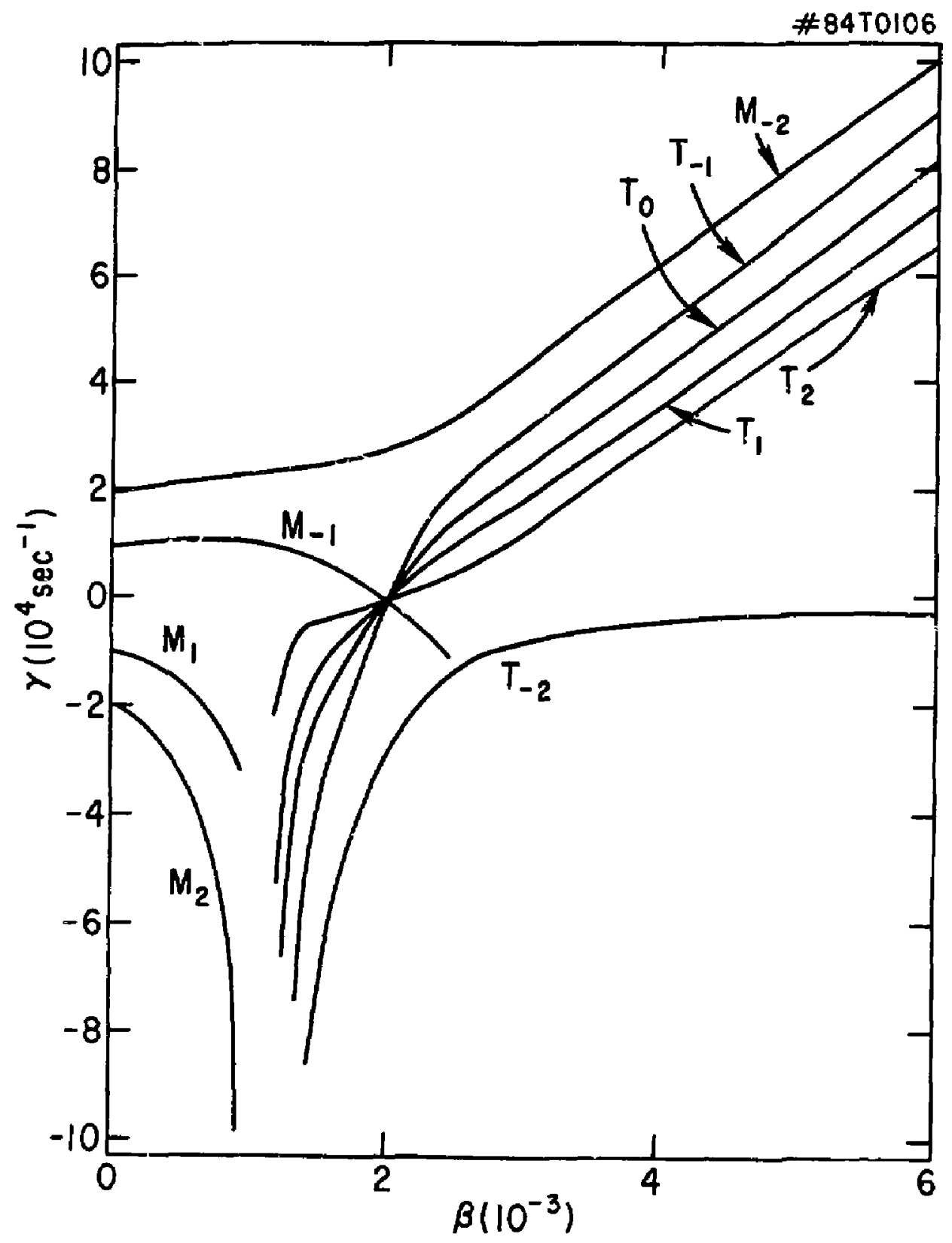

Fig. 5 


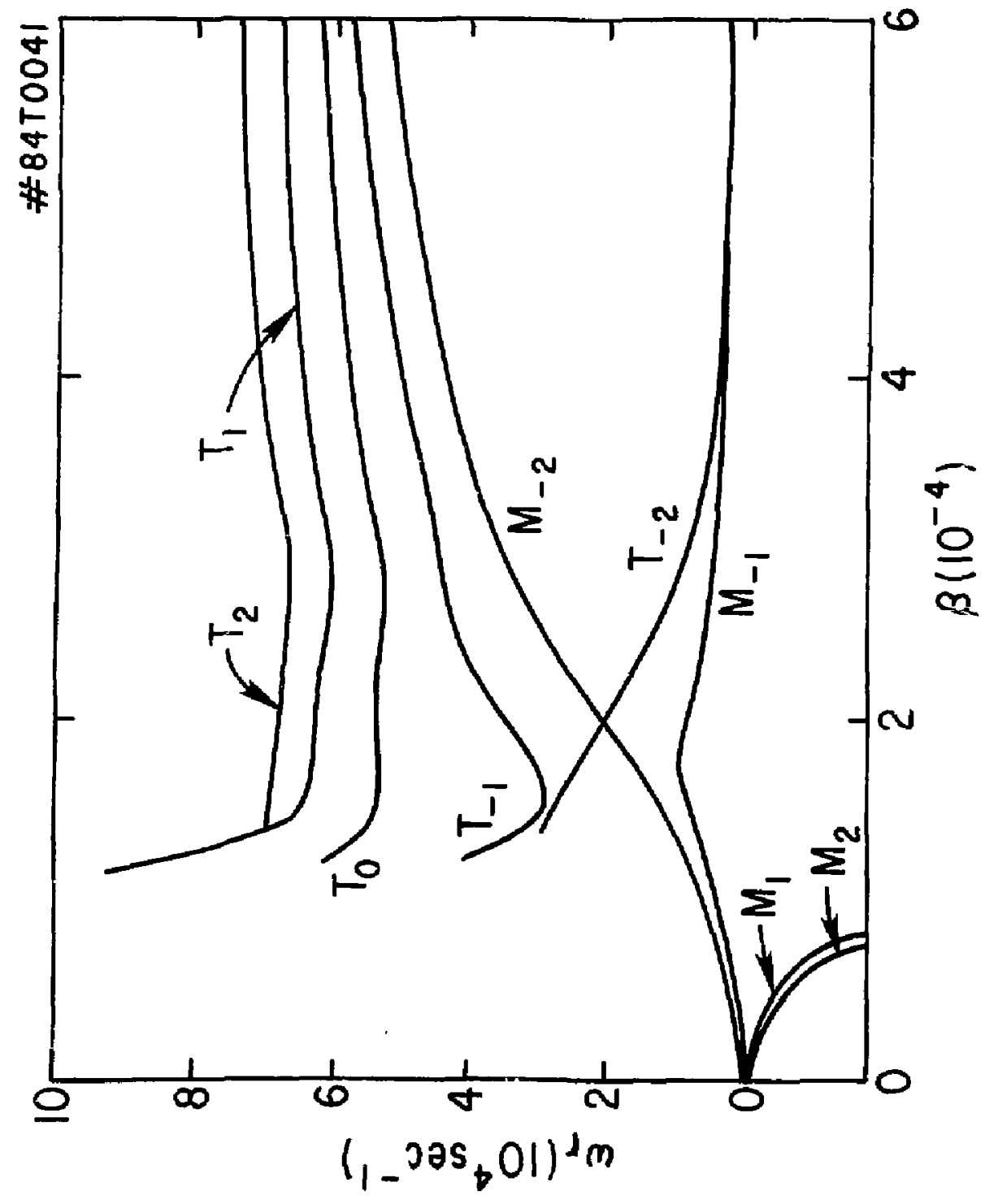




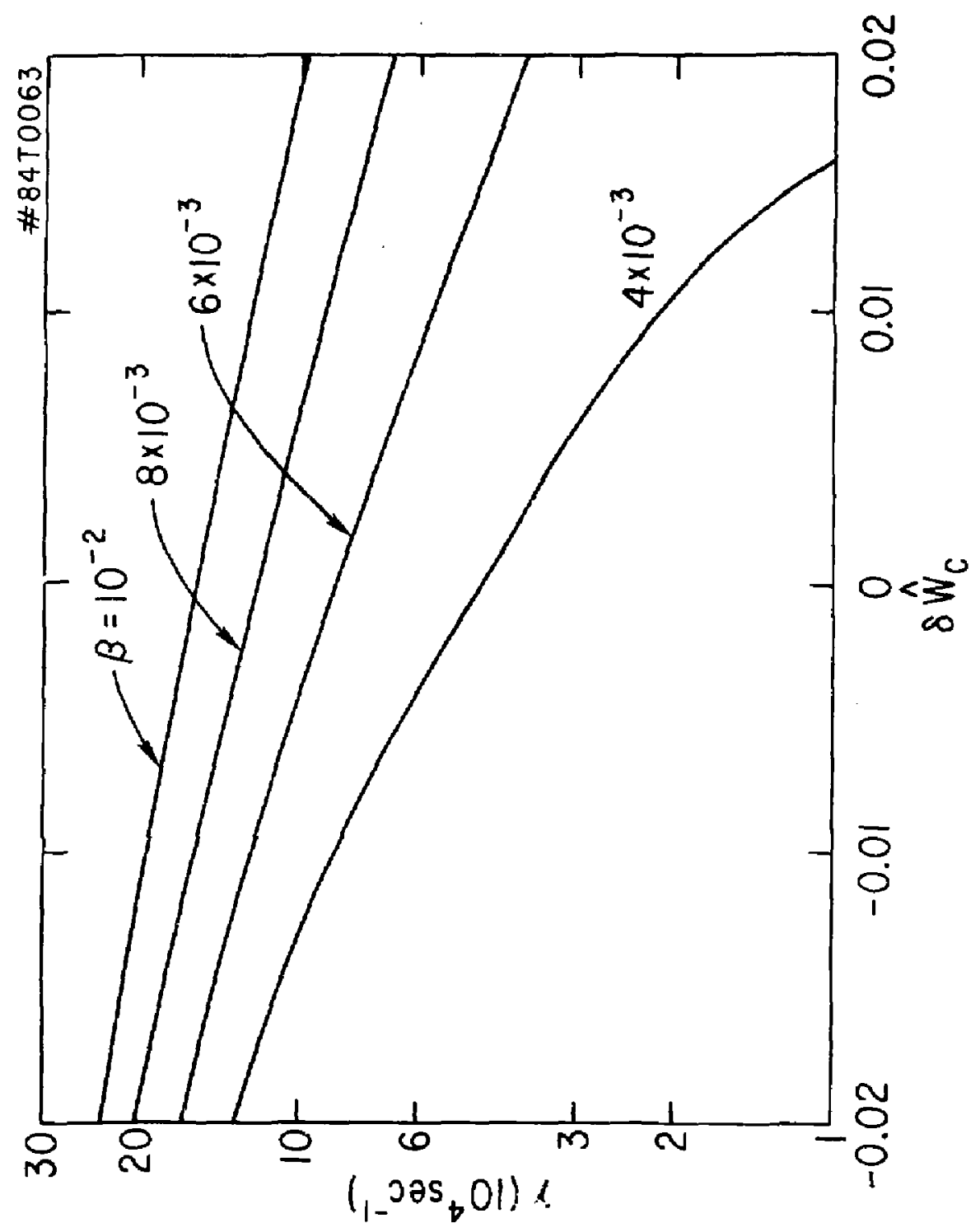




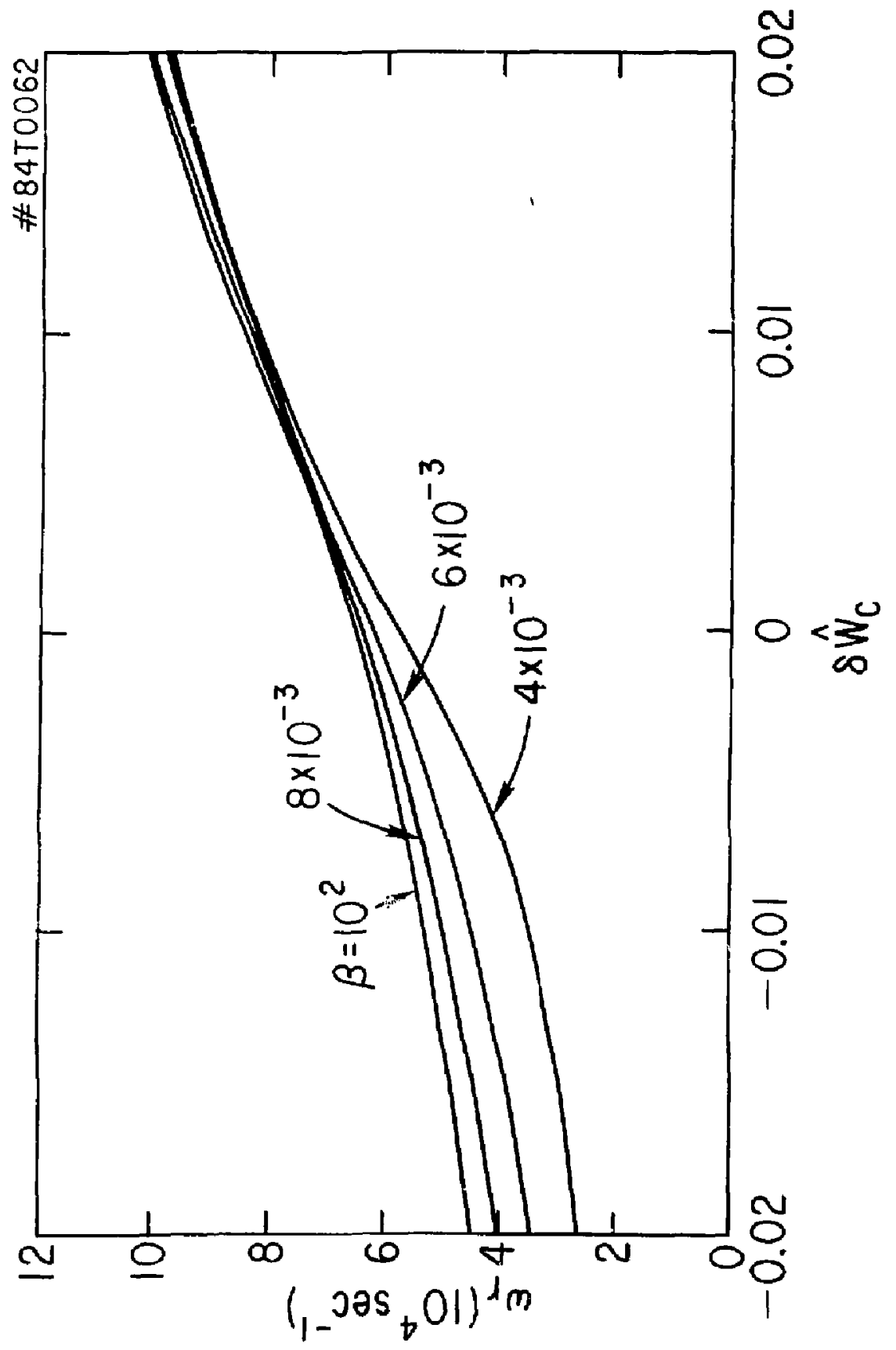

$\stackrel{\infty}{\dot{\sigma}}$ 


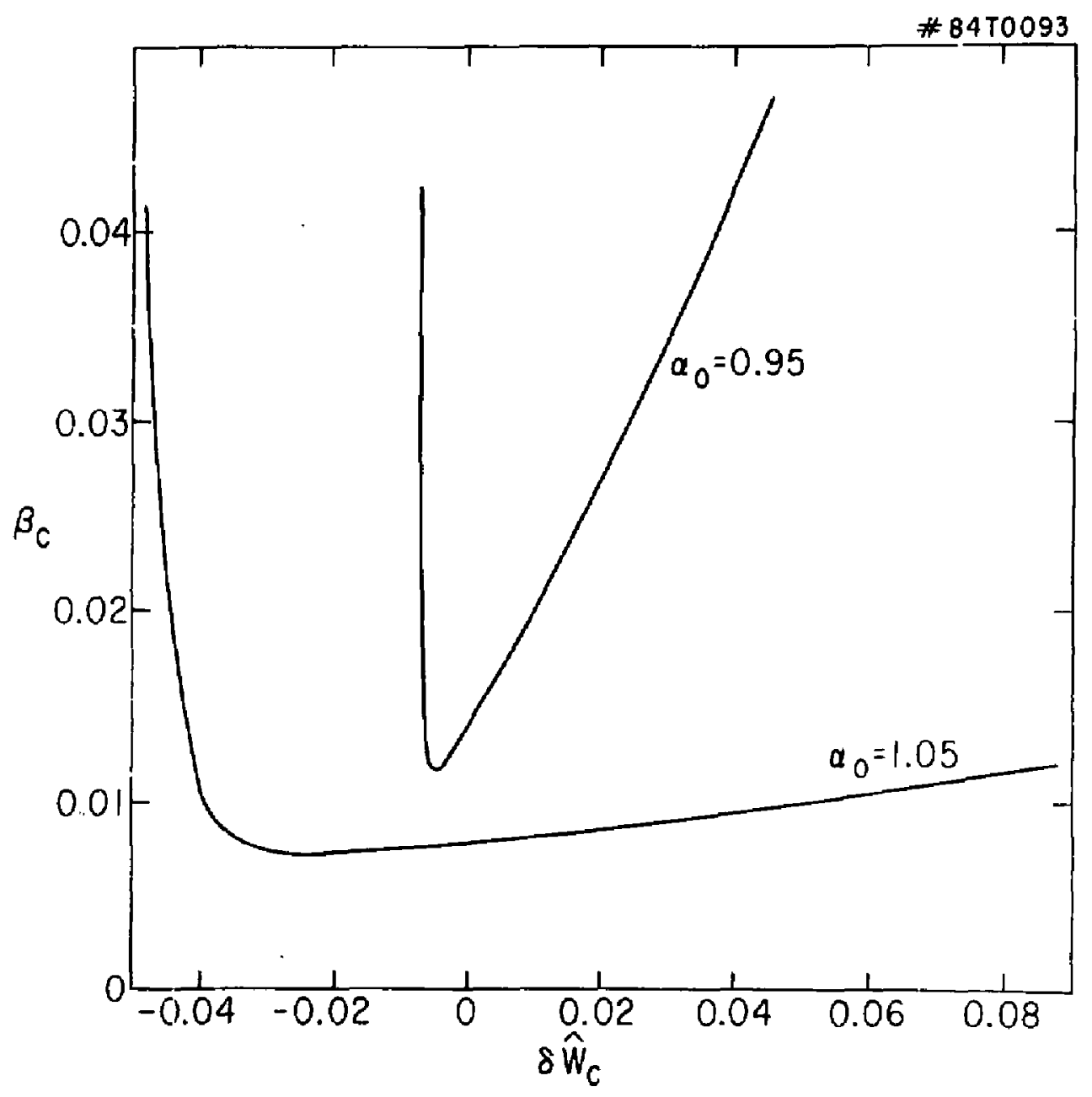

Fig. 9 
Plasmo Res Lab, Austre Net'I Univ, Australia Dr. Frenk J. Paolon1, Unlv of Wollongong, AUSTRALIA Prot. I.R, Jones, Fllinders Unly., AUSTRALIA Frot. H.H. Brennan, Unlr Syoney, AUStral ih Frot. F. Cop, Inst Theo Phys, Austria Frof. Frank Verheest, Inst theoret isehe, BELGIUM Dr. D. Polumbo, Dg XII fuslon Prog, BELGILA Ecole Rayale Milltalre, Lab de Phys PIosmas, BELGium Dr. P.H. Sakanaka, UnIV EstadueI, BRAZIL

Dr. C.R. Jemes, Univ of Alberta, CANADA

Prot. J. Telcnmann, Univ of Montreal, CANADA

Dr. H.M. Skarsgaro, Univ of Saskatchemon, CANAOK

Prof. S.R, Sreenlvason, Unlversity of Calgary, CAkADA

Prof. Tusor $M$, Jonnston, INRS-Energle, CANADa

Dr. Kannes Bernart, Univ Britisn Celumbla, cexpan

Dr. M... Dechynskl, MPB Technologles, Inc., CANhOA

Ehengur LI, Sir Inst Physies, CHINA

Library, Ts ing Huo University, CHIMA

Librarlon, Inst! tute of Physles, CHINA Ins: Plase Pnys, Acosemla SInica, CHINA

Or. Feter Lukse, Komenskeno Univ, ZZEChOSLOVAKIA

The Librartan, Culnari Laboratory. ENGlaho

Prot. Sehaizman, odservatoire de NIce, FRANCE

J. Fudet, Centape, FaAneE

AN DJaes Library, AM Dupas Llbrary, FRAKCE

Dr. Tom Mual, Acudemy B: bliographic, HONG KONG

Preprint Llobrary, Cent Res Inst Phys, Hungary

Dr. :.X. Trehen, Panjab University, INDIA

Dr. Indra, Mahan Lal Das, Banaras Hindu Uniy, INDIA

Dr. L.K. Chavda, South Gujarat Univ, INDIA

Dr. F.K. Chnajlanl, Var Ruent Marg, INDIA

P. Kow, Physlcal Research Lab, InOta

Or. Phillilp Rosenau, I srael inst Tech, ISRAEL

Pfof. 5. Cuperman, Tel Aviv University, ISRaEl

Prof. G, RostagnI, Unly DI Padova, ITALY

Librarian, tht', Ctr Theo Phys, ITALY

wiss Clolis De Palo, Assoc ELRATOM-CNEN, ITALY

BIbliotece, del CNR EURATOM, ITALY

Jr. H. Yamato, Toshibe has l. Dev, JRPAN

Frof. M. Yoshikawa, JAERI, Tekai Res Est, JAPAN

Prot. T. Uenias, University of Tokyo, JaPAN

Reseerch Info Center, Kagoya University. JAPAN

Fraf. Kyojl Nishikawa, Univ of Hiroshjma, JAPAN

Praf. Sigerl Morl, JAERI, JAPAN

Lisrary, Kyoto University, JAPAN

Prot. Ienlro kowakami, Nihan Univ, JAPAN

Frof. Setoshi Itah, Kyusho Universlty, JAPAN

Tech Into Division, Kores Atomlc Energy, KOREA

Dr. R, England, Cluoad Untversitaria, MExico

Bibllotheed, Fonm-lnst voor Plasme, NETHERLANDS

Pro.. B.5. Liley, University of Wolksto, NEW ZEALAND

or. Surast. C. Sharme, Univ ot Colaber, NIGERIA
Prof. J.A.C. Cabrol, Inst Superlor Tech, PORTugal Dr. Oetavitan Fetrus, ALI CLEA University, Romania Prot. M.A. Hellberg, University of Hatal, SO AFRICA Dr. Johon of Yillliers, Atomlc Enargr Bd, SO Africh Fuslon Div, LIbrary, JEN, SPAIN

Prof. Hans hil lhalmson, Cholmers Univ Tech, SWEDEN Dr. Lennart Stentlo, University of LMEA, SWEDEN Llbrary, Royal Inst Tech, SWEDEN

Dr. Erlk T. Kaslson, Uppsala Universitet, SWEDEN Centre de Recherchesen, Ecole Polytech Fed, SWITZERLAhD Or. W.L. Welse, Not'I Bur Stand, USA

Or. W,M. Stacey, Georg Inst Tech, USA

Dr. S,T. Hu, Univ Alobams, USA

Prot. Norman L. Oleson, Univ S Florloo, USA

Dr. Benjatin'm Mo, lowo State Univ, USA

Prot. Magne Kristlonsen. Texas Tecn Unir, LSA

Dr. Roymond Askew, Auburn Uni i , USA

Dr. Y.T. Tolok, Kibrkor Phys Tech ins, LSSR

Dr, D.D. Ryutor, SI berlan Acted 5el, USSR

Dr, G,A, El isoev, Kurehatov Institute, LSSF

Or. Y.A. Glukhikh, Inst Electromhyslcal, USSR

Institute Gor. Physles, Ussh

Prot. T.J. Boyd, Univ Colloge $N$ woles, Wales

Dr. K. Schindler, Ruhr Unlversitat, W, GeMANY Nuclebr Res Estab, Julich Ltd, $N$. GERMANY Llbrarlan, Max-Planck Instltut, W. GEPMANY Dr. H.J. Keoppler, Unlversity Stuttgart, W, GERMANY Blbllothek, Inst Plasmatorsenung, W. Germawy 